\title{
Outcomes Associated with a Screening and Treatment Pathway for Occult Hypoperfusion Following Cardiac Surgery
}

\author{
Ran $\mathrm{Xu}^{1^{*}}$, Greg A. Laine ${ }^{2}$, BeeBee Y. Hu ${ }^{2}$, R. Thomas Solis ${ }^{2}$, Arthur W. Bracey Jr. ${ }^{2}$, \\ James M. Wilson ${ }^{3}$, Amy R. Miclat ${ }^{2}$, Susan Baimbridge ${ }^{3}$, George J. Reul Jr. ${ }^{3}$ \\ ${ }^{1}$ Comprehensive Pharmacy Services, St Luke's The Woodlands Hospital, The Woodlands, USA \\ ${ }^{2}$ St Luke's Episcopal Hospital, Houston, USA \\ ${ }^{3}$ Texas Heart Institute, Houston, USA \\ Email: ${ }^{*}$ rxu@sleh.com
}

Received January 29, 2013; revised March 1, 2013; accepted March 8, 2013

Copyright (C) 2013 Ran Xu et al. This is an open access article distributed under the Creative Commons Attribution License, which permits unrestricted use, distribution, and reproduction in any medium, provided the original work is properly cited.

\begin{abstract}
Introduction: Routinely monitored parameters such as blood pressure (BP) and heart rate may not reliably detect perfusion abnormalities. However, central venous oxygen saturation $\left(\mathrm{ScvO}_{2}\right)$ and lactate levels can detect occult hypoperfusion $(\mathrm{OH})$ and identify patients at risk for complications. The study objective was to assess the impact of an $\mathrm{OH}$ treatment pathway on morbidity and length of stay (LOS) post coronary bypass and valve surgery. Methods: This is a prospective cohort observational study following the implementation of a treatment pathway for $\mathrm{OH}$, defined by $\mathrm{ScvO}_{2}$ $<70 \%$ and lactate $\geq 2 \mathrm{mMol} / \mathrm{L}$ with systolic $\mathrm{BP} \geq 90 \mathrm{mmHg}$. Initial treatment included volume resuscitation and/or blood transfusion, followed by additional interventions when $\mathrm{ScvO}_{2}$ remained $<70 \%$. Repeat lactate was obtained 18 hours postoperatively. Primary outcomes were intensive care unit (ICU)/hospital LOS and complications. Results: Comparing 53 patients managed by the $\mathrm{OH}$ pathway against 21 historical controls, median ICU LOS was 40.4 vs. 49.2 hours $(\mathrm{p}=0.122)$, median hospital LOS 9.2 vs. 11.0 days $(\mathrm{p}=0.0093)$, ICU readmission rate $7.5 \%$ vs. $28.6 \%(\mathrm{p}=$ $0.026)$, and complication rate $26.4 \%$ vs. $47.6 \%(\mathrm{p}=0.101)$. Repeat lactate was checked 18 hours postoperatively in 47 of the 53 patients. Comparing 33 patients with repeat lactate at goal $(<2 \mathrm{mMoL} / \mathrm{L})$ with 14 patients not at goal, median ICU LOS was 35.3 vs. 68.4 hours $(\mathrm{p}=0.061)$, median hospital LOS 8.9 vs. 11.2 days $(\mathrm{p}=0.058)$, median length of mechanical ventilation (LOMV) 13.3 vs. 28.4 hours $(\mathrm{p}=0.0038)$, and complication rate $15.2 \%$ vs. $50.0 \%(\mathrm{p}=0.025)$. Conclusions: An $\mathrm{OH}$ screening and treatment pathway following cardiovascular surgery was associated with significantly shorter hospital LOS and lower ICU readmission rate. Among the OH pathway patients, achieving lactate goal 18 hours postoperatively was associated with significantly shorter LOMV and lower complication rate.
\end{abstract}

Keywords: Occult Hypoperfusion; Cardiovascular Surgery; Lactate; Central Venous Oxygen Saturation; Outcomes

\section{Introduction}

Tissue hypoperfusion is an imbalance between oxygen demand and oxygen delivery. Its origin may be impaired blood oxygenation, blood delivery, hemoglobin concentration or abnormal hemoglobin performance. Prolonged hypoperfusion is associated with organ damage and failure in critical illness [1]. After cardiac surgery, about $10 \%$ of patients experience prolonged intensive care unit (ICU) and hospital length of stay (LOS), predominantly due to organ dysfunction and organ failure [2]. Due to compensatory mechanisms that maintain a normotensive

"Corresponding author. and normoxemic state in the early stages of shock, routinely monitored parameters such as blood pressure, heart rate, arterial oxygen saturation, and urine output may not reflect the adequacy of global perfusion [3,4]. Therefore, additional markers to assess perfusion have been utilized in the critical care setting. Central venous oxygen saturation $\left(\mathrm{ScvO}_{2}\right)$ obtained from the superior vena cava, a wellestablished surrogate for mixed venous oxygen saturation $\left(\mathrm{SvO}_{2}\right)$, reflects the balance between oxygen supply and demand. Previous studies have shown that the difference between $\mathrm{ScvO}_{2}$ and $\mathrm{SvO}_{2}$ is consistently about 5\% across a wide range of cardiorespiratory conditions in both animals and humans $[5,6]$. $\mathrm{ScvO}_{2}$ decreases in a compensa- 
tory fashion when oxygen supply decreases secondary to low cardiac output, hemoglobin or arterial oxygen saturation, or when oxygen demand increases due to fever, shivering, agitation or a hypercatabolic state. When this compensatory process is overwhelmed, global tissue hypoxia and lactic acidosis may ensue, with or without clinical signs of hypoperfusion. $\mathrm{ScvO}_{2}$ and lactate levels obtained during the immediate postoperative period after major general surgery can detect cryptic shock and identify those who may develop complications $[7,8]$. In the cardiac surgery population, lactate levels $>3 \mathrm{mMol} / \mathrm{L}$ in the early postoperative period were associated with increased risk for morbidity and mortality [9]. Maneuvers designed to optimize oxygen delivery in critically ill patients after the development of organ failure have not been shown to improve outcome. However, early recognition and correction of global tissue hypoxia may be beneficial [10]. In severe sepsis, early goal-directed therapy (EGDT) designed to attain an $\mathrm{ScvO}_{2} \geq 70 \%$ during the first 6 hours with volume expansion, packed red blood cells (PRBC) and dobutamine significantly decreased morbidity, mortality, and modulated markers of inflammation and coagulation [11,12]. Furthermore, an EGDT protocol designed to achieve an $\mathrm{SvO}_{2}>70 \%$ and lactate $\leq 2 \mathrm{mMol} / \mathrm{L}$ with volume expansion and dobutamine during the first 8 hours post cardiac surgery decreased morbidity and LOS [13].

In a previous study conducted from October 2007 to June 2008 at our institution, among 60 patients who underwent on-pump aortocoronary bypass and/or valve surgery, 21 patients $(35 \%)$ exhibited occult hypoperfusion, defined as $\mathrm{ScvO}_{2}<70 \%$ and arterial lactate $\geq 2 \mathrm{mMol} / \mathrm{L}$ with a mean arterial pressure $(\mathrm{MAP}) \geq 65 \mathrm{mmHg}$ and urine output $\geq 0.5 \mathrm{~mL} / \mathrm{kg} / \mathrm{hr}$. Patients with severe global tissue hypoxia, defined as $\mathrm{ScvO}_{2}<70 \%$ and lactate $\geq 4$ $\mathrm{mMol} / \mathrm{L}$, did worse compared with those without evidence of global tissue hypoxia. In addition, lactate at 24 hours was a strong predictor of time on mechanical ventilation (MV), Multiple Organ Dysfunction score on days 1, 2, and 7, complications, and ICU/hospital LOS [14]. Based on the results of this study, an occult hypoperfusion screening and treatment pathway was implemented at the same study site. In this follow-up investigation, we proposed that patients undergoing routine screening and treatment for occult hypoperfusion would exhibit improved outcomes, such as shorter ICU and hospital LOS, less time on $\mathrm{MV}$, and lower complication rate compared to patients not treated for occult hypoperfusion.

\section{Methods}

This was a prospective cohort observational study from September 2010 to March 2011 at St. Luke's Episcopal Hospital, a 713-bed academic tertiary care hospital, and home to the Texas Heart Institute. This study was approved by the hospital's institutional review board.

Men and women who were at least 18 years old were eligible for this study if they had aortocoronary bypass or valve surgery requiring cardiopulmonary bypass (CPB) and had $\mathrm{ScvO}_{2}$ and arterial lactate levels obtained upon arrival at the cardiovascular surgery ICU per the occult hypoperfusion screening and treatment pathway. Patients with the following conditions were excluded: cardiogenic shock or arrest prior to surgery, vasopressor therapy prior to surgery, mechanical ventilation prior to surgery, offpump surgery, extracorporeal membrane oxygenation, Jehovah's Witness, intraoperative cardiac arrest, massive perioperative bleeding defined by Thrombolysis In Myocardial Infarction (TIMI) bleeding classification, presence of a pulmonary artery catheter, or presence of a ventricular assist device. All patients were admitted postoperatively to the cardiovascular surgery ICU, and each received standard postoperative care as specified by treating health care teams.

Anesthesia was performed according to institutional practice. Induction was attained with intravenous etomidate. A low-dose opiate anesthetic (fentanyl 10 - $15 \mathrm{mcg}$ / $\mathrm{kg}$ ) was used for maintenance of anesthesia in combination with isoflurane. Rocuronium or cisatracurium was used to achieve neuromuscular blockade. All patients were ventilated mechanically after intubation. Vascular access and monitoring included a radial or brachial artery catheter for measurement of arterial blood pressure and blood sampling, a multilumen internal jugular catheter for measurement of central venous pressure (CVP) and for fluid/medication administration, a Foley catheter to measure urine output, and a nasopharyngeal temperature probe. Two-lead electrocardiogram (leads II and V5) and frontal electroencephalogram were also monitored continuously.

CPB with systemic heparinization (300 units/kg, supplemented with additional boluses to maintain an activated clotting time $>350$ seconds), pump flows from 45 to $55 \mathrm{~mL} / \mathrm{kg} / \mathrm{min}$, moderate systemic hypothermia $\left(28^{\circ} \mathrm{C}\right.$ to $30^{\circ} \mathrm{C}$ ), and intermittent cold blood cardioplegia were used for most patients. Patients were weaned from CPB after the nasopharyngeal temperature reached $36.5^{\circ} \mathrm{C}$. After completion of CPB and removal of cannulae, heparin was reversed with protamine at a 1.5:1 ratio. Intraoperative CPB time and aortic cross-clamp time were obtained for each patient.

After the screening and treatment pathway for occult hypoperfusion was implemented in September 2010, all patients meeting inclusion criteriain whom cardiopulmonary bypass was used, were screened and treated accordingly (Figure 1). $\mathrm{ScvO}_{2}$, arterial lactate, and hemoglobin $(\mathrm{Hgb})$ levels were measured upon arrival at the cardiovascular surgery ICU. Blood pressure, CVP, and 


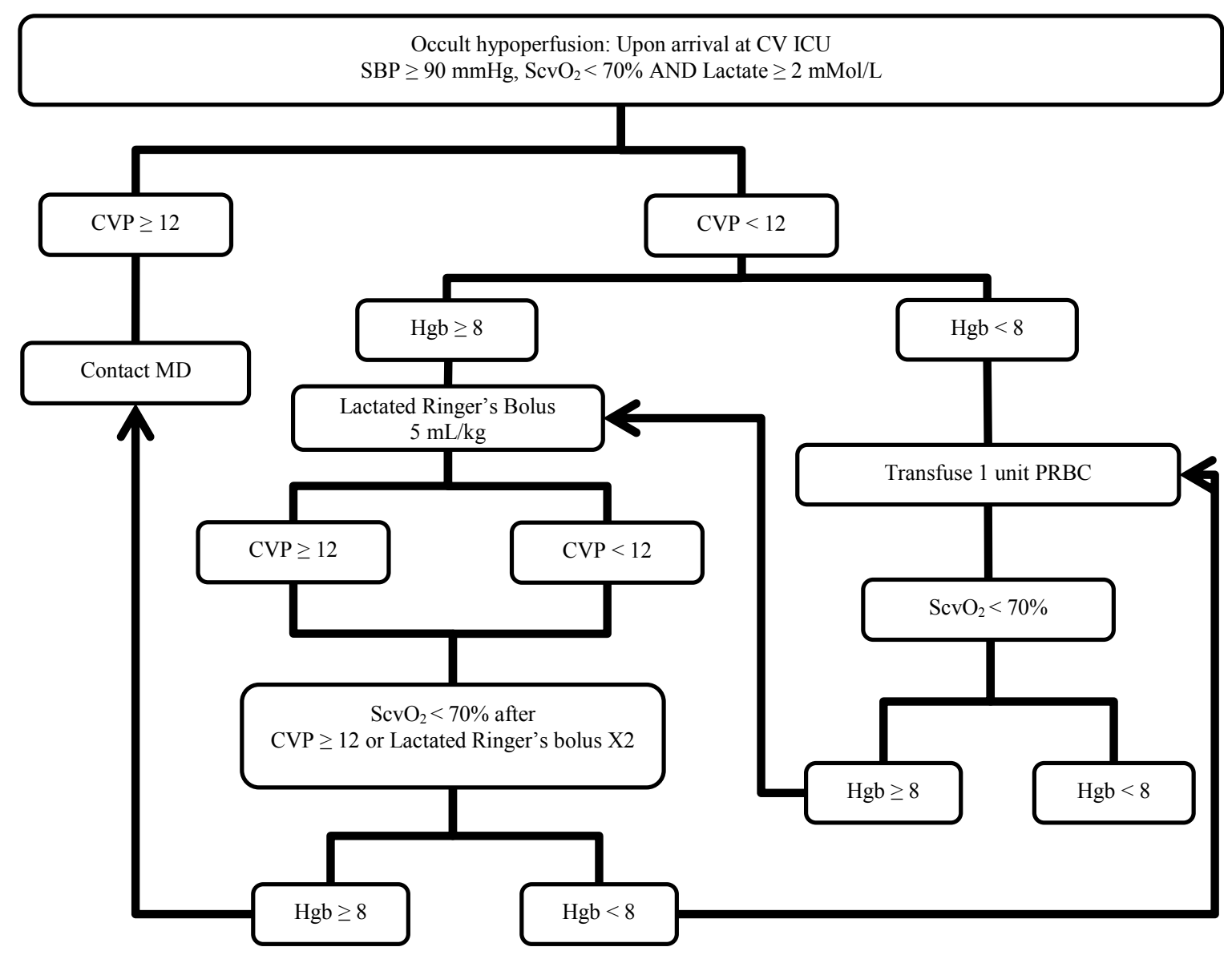

Hemodynamic goals: $\mathrm{CVP} \geq 12 \mathrm{mmHg}, \mathrm{Hgb} \geq 8 \mathrm{~g} / \mathrm{dL}$

Perfusion goals: $\mathrm{ScvO}_{2} \geq 70 \%$ within $6 \mathrm{hr}$, Lactate $<2 \mathrm{mMol} / \mathrm{L}$ at $18 \mathrm{hr}$ after ICU arrival

Figure 1. Occult hypoperfusion screening and treatment pathway; Abbreviations: CV ICU: Cardiovascular Surgery Intensive Care Unit; SBP: Systolic blood pressure; $\mathrm{ScvO}_{2}$ : Central venous oxygen saturation; CVP: Central venous pressure; Hgb: Hemoglobin; PRBC: Packed red blood cells.

other vital signs were monitored continuously. Occult hypoperfusion was identified by $\mathrm{ScvO}_{2}<70 \%$ and arterial lactate $\geq 2 \mathrm{mMol} / \mathrm{L}$ with $\mathrm{SBP} \geq 90 \mathrm{mmHg}$. Initial treatment included volume resuscitation and/or PRBC transfusion to achieve the goals of CVP $\geq 12 \mathrm{mmHg}$, $\mathrm{Hgb} \geq 8 \mathrm{gm} / \mathrm{dL}, \mathrm{ScvO}_{2} \geq 70 \%$, and lactate $<2 \mathrm{mMol} / \mathrm{L}$.

For patients identified with occult hypoperfusion, if CVP $<12 \mathrm{mmHg}$ and $\mathrm{Hgb} \geq 8 \mathrm{gm} / \mathrm{dL}$, Lactated Ringer's (LR) $5 \mathrm{~mL} / \mathrm{kg}$ was administered intravenously over 10 30 minutes and a repeated bolus was allowed once; if $\mathrm{CVP}<12 \mathrm{mmHg}$ and $\mathrm{Hgb}<8 \mathrm{gm} / \mathrm{dL}$, one unit of PRBC was administered, and $\mathrm{Hgb}$ was rechecked. Repeat $\mathrm{ScvO}_{2}$ was obtained one hour after any of the following: CVP $\geq$ $12 \mathrm{mmHg}$, administration of two LR boluses, or after one unit of PRBC transfusion. If repeat $\mathrm{ScvO}_{2}$ remained < $70 \%, \mathrm{Hgb}$ and CVP were rechecked, and the following were performed: If repeat $\mathrm{Hgb}<8 \mathrm{gm} / \mathrm{dL}$ and $\mathrm{CVP}<12$
$\mathrm{mmHg}$, one unit of PRBC was administered; if repeat $\mathrm{Hgb} \geq 8 \mathrm{gm} / \mathrm{dL}$, and CVP $<12 \mathrm{mmHg}$, an LR bolus was administered as above unless a maximum of 2 boluses had already been given. The treating healthcare team was also alerted as patients were identified to facilitate further medical evaluation and individualized treatment if indicated. All off-pathway fluid resuscitation, PRBC usage, inotropic therapy, and vasopressor therapy within 8 hours following admission to the cardiovascular surgery ICU were collected.

Additionally, the treating healthcare team was contacted to evaluate for the need of an inotropic agent, higher CVP or $\mathrm{Hgb}$ target, or other interventions for any of the following: CVP on arrival $\geq 12 \mathrm{mmHg}$ with $\mathrm{ScvO}_{2}$ $<70 \%$ and lactate $\geq 2 \mathrm{mMol} / \mathrm{L}$; repeat $\mathrm{ScvO}_{2}<70 \%$ after $\mathrm{CVP} \geq 12 \mathrm{mmHg}$ attained or repeat $\mathrm{ScvO}_{2}<70 \%$ after 2 LR boluses and/or 2 PRBC transfusions. A repeat arterial 
lactate level was obtained 18 hours after the initial measurement (Figure 1).

The outcomes of eligible patients undergoing the screening and treatment pathway for occult hypoperfusion were compared with historical controls prior to the implementation of the pathway. This control group was from our previous study conducted from 2007-2008, where treating physicians and other healthcare providers were blinded to the $\mathrm{ScvO}_{2}$ and lactate results [14]. In addition, among the patients managed by the occult hypoperfusion pathway, those achieving the secondary resuscitation goal of repeat lactate $<2 \mathrm{mMol} / \mathrm{L}$ following treatment were compared with those not achieving this goal.

The primary outcome of this study was ICU and hospital LOS in patients managed by the occult hypoperfusion pathway compared with historical controls prior to the implementation of the pathway. Secondary outcomes included ICU readmission rate, length of mechanical ventilation and complication rate. Postoperative complications included development of renal dysfunction or failure defined as a serum creatinine increase $>177 \mu \mathrm{mol} / \mathrm{L}$, serum creatinine increase $>50 \%$, or dialysis requirement; prolonged ventilation defined as length of mechanical ventilation $>48$ hours; cardiogenic shock or cardiac arrest; development of acute respiratory distress syndrome, sepsis or infection. All complications were defined before commencement of the trial and were diagnosed and managed by non-research staff.

The Kolmogorov-Smirnov test was conducted to test the normality of continuous data. Student's t-test was used to analyze normally distributed continuous data, the
Mann Whitney U-test was used for continuous data that failed normality testing, and Fisher's exact test was used for categorical data. All tests were two-tailed, and a $\mathrm{p}$ value of less than 0.05 was deemed statistically significant. Statistical analysis was performed using GraphPad Prism 6 (San Diego, CA).

\section{Results}

A total of 479 on-pump cardiac surgery cases were screened; 89 patients were excluded due to procedures other than on-pump aortocoronary bypass or valve surgery, having a pulmonary artery catheter, on extracorporeal membrane oxygenation or with incomplete data. Among the 390 cases evaluated, 15 patients were further excluded secondary to a postoperative systolic blood pressure $<90 \mathrm{mmHg}$ upon arrival to the cardiovascular surgery ICU. Among the remaining 375 cases, 120 cases had normal $\mathrm{ScvO}_{2}(\geq 70 \%)$ and arterial lactate $(<2$ $\mathrm{mMol} / \mathrm{L}) ; 112$ cases had isolated low $\mathrm{ScvO}_{2}(<70 \%) ; 90$ had isolated high lactate $(\geq 2 \mathrm{mMol} / \mathrm{L})$; and the remaining 53 cases were classified as occult hypoperfusion, with $\mathrm{ScvO}_{2}<70 \%$, arterial lactate $\geq 2 \mathrm{mMol} / \mathrm{L}$ and a systolic blood pressure $\geq 90 \mathrm{mmHg}$ (Figure 2). None of the 53 patients exhibited oliguria (urine output $<0.5 \mathrm{~mL} /$ $\mathrm{kg} / \mathrm{hr}$ ) upon cardiovascular surgery ICU arrival with the exception of one patient with anuria secondary to pre-existing dialysis-dependent end stage renal disease. These 53 patients were compared with the historical controls of 21 occult hypoperfusion patients following aortocoronary bypass and/or valve surgery from our previous study [14].

Thirty-nine of the 53 patients had a CVP $<12 \mathrm{mmHg}$

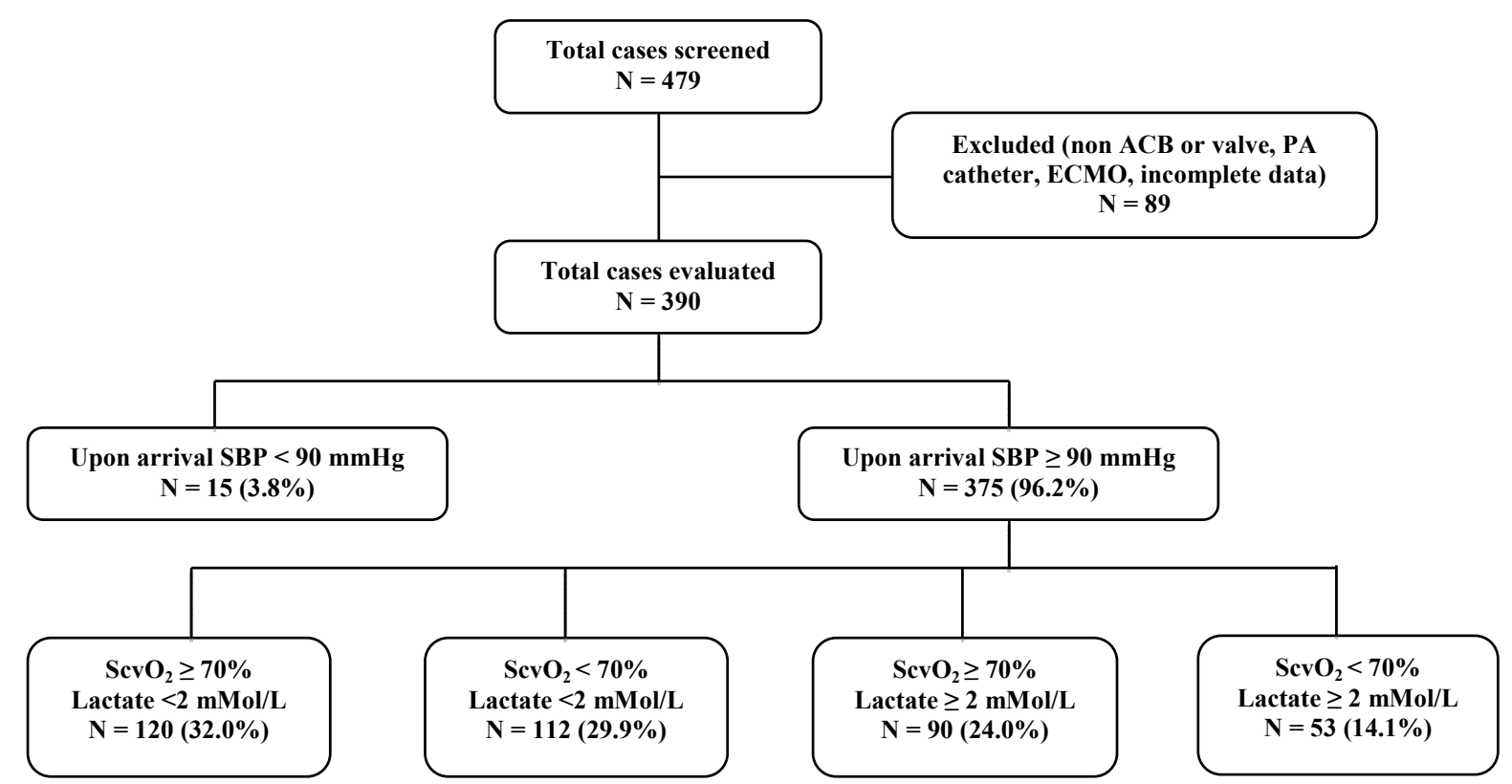

Figure 2. Patient distribution; Abbreviations: ACB: Aortocoronary bypass; PA: Pulmonary artery; ECMO: Extracorporeal membrane oxygenation; SBP: Systolic blood pressure; $\mathrm{ScvO}_{2}$ : Central venous oxygen saturation. 
upon arrival to the cardiovascular surgery ICU. Treatment was initiated in 33 patients $(84.6 \%)$. The treatment pathway (LR and/or PRBC per Figure 1) was initially utilized in 24 cases, supplemental resuscitation (additional crystalloid, albumin, PRBC or fresh frozen plasma) was ordered in addition to the pathway in 10 of these 24 cases, and individualized off-pathway resuscitation (albumin or fresh frozen plasma) was ordered in the other 9 cases. The mean resuscitation volume initially administered to these 33 patients was $1880 \pm 1423 \mathrm{~mL}$ in crystalloid equivalents (range: $500 \mathrm{~mL}$ to $6200 \mathrm{~mL}$ ), assuming $1 \mathrm{~mL}$ of colloid $=3 \mathrm{~mL}$ of crystalloid. A repeat $\mathrm{ScvO}_{2}$ measurement was obtained in 24 of the 33 patients following initial resuscitation. However, the repeat $\mathrm{ScvO}_{2}$ reached goal in only four of the 24 patients. Of the 20 patients not achieving the $\mathrm{ScvO}_{2}$ goal of $\geq 70 \%, 12$ patents received additional off-pathway fluid resuscitation, PRBC and/or inotropic therapy. Of the 14 patients initially presenting to the cardiovascular surgery ICU with a $\mathrm{CVP} \geq 12 \mathrm{mmHg}, 6$ patients received individualized offpathway fluid resuscitation, $\mathrm{PRBC}$ and/or inotropic therapy. Overall, the CVP goal of $\geq 12 \mathrm{mmHg}$ was achieved in $41(77.4 \%)$ out of 53 patients.

Repeat arterial lactate levels were obtained 18 hours postoperatively in 47 of the 53 patients. Thirty-three $(70.2 \%)$ of 47 patients achieved the resuscitation goal of arterial lactate $<2 \mathrm{mMol} / \mathrm{L}$, and the remaining 14 (29.8\%) patients had an arterial lactate $\geq 2 \mathrm{mMol} / \mathrm{L}$ at the 18 hour time point. Of the 33 patients treated for a CVP $<12$ $\mathrm{mmHg}$, the percentage of patients achieving the lactate goal was highest in those where the resuscitation pathway was solely utilized (11 of 14 patients, $78.6 \%$ ), followed by those receiving supplemental resuscitation in addition to the pathway ( 7 of 10 patients, $70 \%$ ), followed by those receiving only individualized off-pathway resuscitation ( 5 of 9 patients, 55.6\%). However, none of these differences were statistically significant.
Baseline characteristics of the 53 patients managed by the occult hypoperfusion pathway (post-implementation group) and the 21 historical controls prior to the implementation of the occult hypoperfusion pathway (preimplementation group) are listed in Table 1. Age, percent male, percent diabetic, cardiopulmonary bypass time, aortic cross clamp time, postoperative $\mathrm{ScvO}_{2}$, postoperative arterial lactate, and EuroSCORE [15] were compared between the two groups. Data collected for all of the above continuous parameters failed the KolmogorovSmirnov test for normality except age. Therefore, nonparametric tests were utilized. No significant differences were found between the two groups (Table 1).

The primary outcome of hospital LOS among patients in the post-implementation group was significantly shorter compared with the pre-implementation group (median 9.2 days vs. 11.0 days, $\mathrm{p}=0.0093$ ). However, no significant difference in ICU LOS was found between the 2 groups (median 40.4 hours vs. 49.2 hours, $p=0.122$ ). For secondary outcomes, patients in the post-implementation group had a significantly lower ICU readmission rate compared with those in the pre-implementation group $(7.5 \%$ vs. $28.6 \%, p=0.026)$. Length of mechanical ventilation and complication rate were not significantly different between the two groups. However, median number of complications per patient was significantly lower in the post-implementation group (Table 2).

Among patients in the post-implementation group, those who had a repeat arterial lactate $<2 \mathrm{mMol} / \mathrm{L}$ were compared with those with a repeat lactate $\geq 2 \mathrm{mMol} / \mathrm{L}$. Among patients achieving the lactate goal of $<2 \mathrm{mMol} / \mathrm{L}$, there was a trend towards shorter ICU LOS (median 35.3 hours vs. 68.4 hours, $\mathrm{p}=0.061$ ) and shorter hospital LOS (median 8.9 days vs. 11.2 days, $\mathrm{p}=0.058$ ). Additionally, length of mechanical ventilation was significantly shorter (median 13.3 hours vs. 28.4 hours, $\mathrm{p}=0.0038$ ); complication rate was significantly lower $(15.2 \%$ vs. $50.0 \%, \mathrm{p}=$

Table 1. Comparative baseline characteristics.

\begin{tabular}{|c|c|c|c|c|}
\hline Parameter & Pre-implementation $(n=21)$ & Post-implementation $(n=53)$ & p value & 95\% Confidence interval \\
\hline Age (mean, SD, yr) & $66.0 \pm 11.1$ & $63.3 \pm 13.2$ & $0.368^{*}$ & -3.745 to $9.236^{*}$ \\
\hline Sex (\% male) & 67 & 53 & $0.310^{\S}$ & - \\
\hline Diabetes (\%) & 42.8 & 41.5 & $1.0^{\S}$ & - \\
\hline $\begin{array}{l}\text { Cardiopulmonary bypass time } \\
\text { (median, IQR, min) }\end{array}$ & $68.0(53.5-131.5)$ & $68.0(48.5-130.5)$ & $0.838^{\dagger}$ & - \\
\hline $\begin{array}{l}\text { Aortic cross clamp time } \\
\text { (median, IQR, min) }\end{array}$ & $44.0(36.0-80.0)$ & $54.0(35.5-83.0)$ & $0.834^{\dagger}$ & - \\
\hline $\mathrm{ScvO}_{2}$ (median, IQR, \%) & $64.5(56.0-66.3)$ & $64.7(60.0-68.2)$ & $0.177^{\dagger}$ & - \\
\hline Lactate (median, IQR, mMol/L) & $3.0(2.3-4.3)$ & $2.6(2.2-3.1)$ & $0.103^{\dagger}$ & - \\
\hline EuroSCORE (median, IQR) & $6.0(2.5-8.0)$ & $5.0(3.5-7.0)$ & $0.796^{\dagger}$ & - \\
\hline
\end{tabular}

${ }^{*} \mathrm{p}$ value and $95 \%$ confidence interval calculated by unpaired t-test (normal distribution). ${ }^{\S} \mathrm{p}$ value calculated by Fisher's exact test. ${ }^{\dagger} \mathrm{p}$ value calculated by MannWhitney U test (for non-normal distribution). 
Table 2. Comparative outcomes: pre-implementation (historical controls) versus post-implementation of the occult hypoperfusion screening and treatment pathway.

\begin{tabular}{lccc}
\hline Parameter & Pre-implementation (n= 21) & Post-implementation (n= 53) $^{\text {p value }}$ \\
\hline ICU LOS (median, IQR, hours) & $49.2(37.4-108.5)$ & $40.4(30.4-69.6)$ & 0.122 \\
Hospital LOS (median, IQR, days) & $11.0(9.0-21.0)$ & $9.2(7.4-14.1)$ & 0.0093 \\
ICU Readmission Rate (\%) & 28.6 & 7.5 & 0.026 \\
Length of Mechanical Ventilation (median, IQR, hours) & $11.7(7.5-26.0)$ & $14.8(9.5-23.4)$ & 0.210 \\
Complication Rate (\%) & 47.6 & 26.4 & 0.101 \\
Number of Complications per Patient (median, IQR) & $0.0(0.0-2.0)$ & $0.0(0.0-1.0)$ & 0.044 \\
\hline
\end{tabular}

IQR: Interquartile range; ${ }^{\mathrm{p}} \mathrm{p}$ value calculated by Mann Whitney $\mathrm{U}$ test for continuous data and Fisher's exact test for categorical data.

Table 3. Comparative outcomes post-implementation of the occult hypoperfusion screening and treatment pathway: followup lactate at goal $(<2 \mathrm{mMol} / \mathrm{L})$ versus not at goal $(\geq 2 \mathrm{mMol} / \mathrm{L})$.

\begin{tabular}{lccc}
\hline \multicolumn{1}{c}{ Parameter } & Follow-up lactate at goal $(\mathbf{n}=\mathbf{3 3})$ & Follow-up lactate NOT at goa $(\mathbf{n}=\mathbf{1 4})$ & $\mathbf{p}$ value \\
\hline ICU LOS (median, IQR, hours) & $35.3(29.6-50.6)$ & $68.4(36.3-195)$ & 0.061 \\
Hospital LOS (median, IQR, days) & $8.9(7.3-12.4)$ & $11.2(9.2-16.1)$ & 0.058 \\
ICU Readmission Rate (\%) & 9.1 & 7.1 & 1.000 \\
$\begin{array}{l}\text { Length of Mechanical Ventilation } \\
\text { (median, IQR, hours) }\end{array}$ & $13.3(8.8-21.4)$ & $28.4(15.8-104.3)$ & 0.0038 \\
$\begin{array}{l}\text { Complication Rate (\%) } \\
\text { Number of Complications per Patient } \\
\text { (median, IQR) }\end{array}$ & 15.2 & 50 & 0.025 \\
\hline
\end{tabular}

IQR: Interquartile range; " $\mathrm{p}$ value calculated by Mann Whitney U test for continuous data and Fisher's exact test for categorical data.

0.025 ) and number of complications per patient was significantly lower (median 0.0 vs. $0.5, \mathrm{p}=0.011$ ). No significant difference between the 2 groups was found in ICU readmission rate (Table 3). When compared to the historical control group, patients achieving the lactate goal of $<2 \mathrm{mMol} / \mathrm{L}$ at the 18 hour time point also experienced a significantly shorter ICU LOS (median 35.3 hours vs. 49.2 hours, $p=0.046$ ), hospital LOS (median 8.9 days vs. 11.0 days, $\mathrm{p}=0.001$ ), complication rate $(15.2 \%$ vs. $47.6 \%, p=0.014)$ and complications per patient (median 0.0 vs. $0.0, \mathrm{p}=0.005$ ); mean difference was 1 complication per patient).

\section{Discussion}

We have suggested that the early treatment of occult hypoperfusion following on-pump cardiac surgery reduces hospital LOS, ICU readmission rate, and complications per patient. Furthermore, among the patients managed by the occult hypoperfusion pathway, achieving the lactate goal after resuscitation was associated with significantly shorter length of mechanical ventilation and lower complication rate, as well as trends towards shorter hospital and ICU LOS. This is consistent with the hypothesis that hypoperfusion evading clinical observation is clinically important and may impact ICU stay duration and patient outcome. These observations warrant confirmation and consideration for routine monitoring on all operated patients with heart disease that may impact their hemodynamics. In studies of high risk general surgery patients, abnormally low $\mathrm{ScvO}_{2}$ in the perioperative period was associated with increased incidence of postoperative complications. Pearse and colleagues [8] identified the lowest $\mathrm{ScvO}_{2}$ during the early postoperative period as an independent predictor of postoperative complications. The authors observed a considerable decrease in $\mathrm{ScvO}_{2}$ within the first hours after surgery, and the optimal cutoff value for the lowest $\mathrm{ScvO}_{2}$ was $64.4 \%$. The Collaborative Study Group on Perioperative $\mathrm{ScvO}_{2}$ Monitoring [16] found that $\mathrm{ScvO}_{2}$ was reduced at various time points during the perioperative period in patients who developed complications. The cut-off value for mean $\mathrm{ScvO}_{2}$ was $73 \%$ in this study. In addition, Ranucci et al. [17] reported that the combined index of $\mathrm{ScvO}_{2}<68 \%$ and lactate $>3 \mathrm{mMol} / \mathrm{L}$ intraoperatively predicted major morbidity after cardiac surgery in pediatric patients, with a positive predictive value of $89 \%$. Similarly, $\mathrm{Hu}$ et al. [14] also found that the combination of low $\mathrm{ScvO}_{2}$ and high lactate upon ICU arrival was associated with increased morbidity in the adult cardiac surgery population.

Although there are an abundance of observational 
studies reporting that abnormalities in various measures addressing oxygen transport are predictive of poor outcome, there are few interventional studies using the same measures as therapeutic targets. A prospective, randomized, controlled trial conducted by Donati et al. [18] demonstrated that early treatment directed to maintain an oxygen extraction ratio (derived from $\mathrm{ScvO}_{2}$ ) $<27 \%$ reduced organ failures and hospital LOS in high risk abdominal surgery patients. In an earlier non-blinded randomized controlled trial, Polonen et al. [13] utilized a goal-oriented protocol to maintain the target of $\mathrm{SvO}_{2}>$ $70 \%$ and lactate $<2 \mathrm{mMol} / \mathrm{L}$ in post cardiac surgery patients. The median hospital LOS was significantly shorter, and morbidity was less frequent in the protocol group compared with the control group. However, not all patients presented with low $\mathrm{SvO}_{2}$ and/or elevated lactate upon ICU arrival. The incidence of overt versus occult hypoperfusion was not described, and additional parameters derived from the pulmonary artery catheter such as cardiac index and pulmonary capillary wedge pressure were utilized to guide resuscitation. In comparison, our study focused specifically on patients presenting with occult hypoperfusion upon ICU arrival (those with normal blood pressure but abnormal perfusion parameters). Additionally, we used $\mathrm{ScvO}_{2}$ instead of $\mathrm{SvO}_{2}$ as one of the markers for hypoperfusion, since most of the cardiac surgery patients at our institution are not managed with a pulmonary artery catheter.

Goal-directed therapy using other markers that require more invasive monitoring has been studied in surgical patients. Goepfert et al. demonstrated in cardiac surgery patients that a goal-directed fluid management strategy based on global end-diastolic volume index (GEDVI) led to reduced need for vasopressors, catecholamines, mechanical ventilation, and ICU therapy compared to a historical control group. The measurement of GEDVI was helpful for reliably assessing central blood flow using a transcardiopulmonary thermodilution technique, but required the insertion of a special catheter prior to surgery [19]. In another prospective, randomized trial among high-risk patients undergoing major elective surgeries, pulmonary artery catheter-guided optimization of oxygen delivery index $\left(\mathrm{DO}_{2} \mathrm{I}\right)$ was studied using fluids alone vs. fluids and dobutamine, during the intraoperative and 24 hour postoperative periods. The authors found that the use of dobutamine was associated with better outcomes [20].

One of the limitations of our study was the inclusion of historical controls. However, the 2 groups were comparable in terms of preoperative EuroSCORE, incidence of diabetes, CPB time, and aortic cross-clamp time, factors known to be associated with development of global tissue hypoxia [14]. At the study site, the only change to anesthesia technique that occurred between 2008 and
2010 was the substitution of rocuronium or cisatracurium for maintenance of muscle relaxation due to the pancuronium shortage. It is unlikely that this change would affect the results of this study. Other than the occult hypoperfusion screening and treatment pathway, no additional changes to postoperative management were introduced.

The fact that a low percentage of patients achieved a repeat $\mathrm{ScvO}_{2} \geq 70 \%$ after initial resuscitation in this study suggests that a more aggressive strategy may be necessary and explain why a subset of patients failed to normalize lactate at the 18 hour time point. A follow-up $\mathrm{ScvO}_{2}$ was only performed once shortly after fluid boluses or RBC transfusion, and it is unknown what percentage of patients eventually achieved the $\mathrm{ScvO}_{2}$ goal. Assessing lactate at an earlier time point may be warranted as well. We recently began rechecking lactate at 4 hours rather than 18 hours postoperatively to more quickly identify patients that may require additional medical evaluation and/or treatment. Additionally, compliance with the occult hypoperfusion pathway was not $100 \%$, as this study was not conducted in a highly controlled research environment. However, it reflected a more realistic clinical practice setting, and the improved outcomes may have resulted in part from earlier identification of patients with perfusion abnormalities that cannot be detected by alterations in vital signs, urine output or other routinely monitored parameters.

In conclusion, implementation of a screening and treatment pathway for occult hypoperfusion in cardiac surgery patients without the use of a pulmonary artery catheter or other advanced monitoring device was associated with improved clinical outcomes. Furthermore, achieving the repeat lactate goal post treatment was associated with better morbidity outcomes. More aggressive resuscitation and earlier assessment of lactate clearance may benefit selected patients with occult hypoperfusion following cardiac surgery.

\section{REFERENCES}

[1] G. Friedman, D. De Backer, M. Shahla, et al., "Oxygen Supply Dependency Can Characterize Septic Shock," Intensive Care Medicine, Vol. 24, No. 2, 1998, pp. 118-123. doi:10.1007/s001340050531

[2] T. A. Ryan, M. Y. Rady, C. A. Bashour, et al., "Predictors of Outcome in Cardiac Surgical Patients with Prolonged Intensive Care Stay," Chest, Vol. 112, No. 4, 1997, pp. 1035-1042. doi:10.1378/chest.112.4.1035

[3] W. C. Shoemaker, C. Wo, D. Thangathurai et al., "Hemodynamic Patterns of Survivors and Nonsurvivors during High Risk Elective Surgical Operations," World Journal of Surgery, Vol. 23, No. 12, 1999, pp. 1264-1271. doi: $10.1007 / \mathrm{s} 002689900660$

[4] M. Y. Rady, E. P. Rivers and R. M. Nowak, "Resuscita- 
tion of the Critically Ill in the ED: Responses of Blood Pressure, Heart Rate, Shock Index, Central Venous Oxygen Saturation and Lactate," American Journal of Emergency Medicine, Vol. 14, No. 2, 1996, pp. 218-225. doi:10.1016/S0735-6757(96)90136-9

[5] K. Reinhart, T. Rudolph, D. L. Bredle, et al., "Comparison of Central Venous to Mixed Venous Oxygen Saturation during Changes in Oxygen Supply/Demand," Chest, Vol. 95, No. 6, 1989, pp. 1216-1221. doi: $10.1378 /$ chest.95.6.1216

[6] J. C. Berridge, "Influence of Cardiac Output on the Correlation between Mixed Venous and Central Venous Oxygen Saturation," British Journal of Anaesthesia, Vol. 69, No. 4, 1992, pp. 409-410. doi:10.1093/bja/69.4.409

[7] A. Meregalli, R. P. Oliveira and G. Friedman, "Occult Hypoperfusion Is Associated with Increased Mortality in Hemodynamically Stable, High-Risk Surgical Patients," Critical Care, Vol. 8, 2004, pp. 60-65. doi:10.1186/cc2423

[8] R. Pearse, D. Dawson, J. Fawcett, et al., "Changes in Central Venous Saturation after Major Surgery, and Association with Outcome," Critical Care, Vol. 9, 2005, pp. 694-699. doi:10.1186/cc3888

[9] J. Maillet, P. Le Besnerais, M. Cantoni, et al., "Frequency, Risk Factors, and Outcome of Hyperlactatemia after Cardiac Surgery," Chest, Vol. 123, No. 5, 2003, pp. 13611366. doi:10.1378/chest.123.5.1361

[10] J. W. Kern and W. C. Shoemaker, "Meta-Analysis of Hemodynamic Optimization in High-Risk Patients," Critical Care Medicine, Vol. 30, No. 8, 2002, pp. 1686-1692. doi:10.1097/00003246-200208000-00002

[11] E. Rivers, B. Nguyen, S. Havstad, et al., "Early GoalDirected Therapy in the Treatment of Severe Sepsis and Septic Shock," New England Journal of Medicine, Vol. 345, No. 19, 2001, pp. 1368-1377. doi:10.1056/NEJMoa010307

[12] R. M. Otero, B. Nguyen, D. T. Huang, et al., "Early GoalDirected Therapy in Severe Sepsis and Septic Shock Revisited," Chest, Vol. 130, No. 5, 2006, pp. 1579-1595. doi:10.1378/chest.130.5.1579
[13] P. Polonen, E. Roukonen, M. Hippelainen, et al., "A Prospective, Randomized Study of Goal-Oriented Hemodynamic Therapy in Cardiac Surgical Patients," Anesthesia \& Analgesia, Vol. 90, No. 5, 2000, pp. 1052-1059. doi:10.1097/00000539-200005000-00010

[14] B. Y. Hu, G. A. Laine, S. Wang, et al., "Combined Central Venous Oxygen Saturation and Lactate as Markers of Occult Hypoperfusion and Outcome Following Cardiac Surgery," Journal of Cardiothoracic and Vascular Anesthesia, Vol. 26, No. 1, 2012, pp. 52-57. doi:10.1053/j.jvca.2011.07.021

[15] S. A. Nashef, F. Roques, P. Michel, E. Gauducheau, S. Lemeshow and R. Salamon, "EuroSCORE European System for Cardiac Operative Risk Evaluation (EuroSCORE)," European Journal Cardio-Thoracic Surgery, Vol. 16, No. 1, 1999, pp. 9-13. doi:10.1016/S1010-7940(99)00134-7

[16] Collaborative Study Group on Perioperative $\mathrm{ScvO}_{2} \mathrm{Mo-}$ nitoring, "Multicentre Study on Peri- and Postoperative Central Venous Oxygen Saturation in High-Risk Surgical Patients," Critical Care, Vol. 10, No. 6, 2006, p. R158.

[17] M. Ranucci, G. Isgrò, C. Carlucci, et al., "Surgical and Clinical Outcome Research Group. Central Venous Oxygen Saturation and Blood Lactate Levels during Cardiopulmonary Bypass Are Associated with Outcome after Pediatric Cardiac Surgery," Critical Care, Vol. 14, 2010, p. R149.

[18] A. Donati, S. Loggi, J. C. Preiser, et al., "Goal-Directed Intraoperative Therapy Reduces Morbidity and Length of Hospital Stay in High-Risk Surgical Patients," Chest, Vol. 132, No. 6, 2007, pp. 1817-1824. doi:10.1378/chest.07-0621

[19] M. S. Goepfert, D. A. Reuter, D. Akyol, et al., "GoalDirected Fluid Management Reduces Vasopressor and Catecholamine Use in Cardiac Surgery Patients," Intensive Care Medicine, Vol. 33, No. 1, 2007, pp. 96-103. doi:10.1007/s00134-006-0404-2

[20] S. M. Lobo, F. R. Lobo, C. A. Polachini, et al., "Prospective, Randomized Trial Comparing Fluids and Dobutamine Optimization of Oxygen Delivery in High-Risk Surgical Patients," Critical Care, Vol. 10, 2006, p. R72. doi:10.1186/cc4913 